\title{
Multisensory stimulation and individualized music sessions on older adults with severe dementia: effects on mood, behavior, and biomedical parameters
}

\author{
Ana Maseda, Nuria Cibeira, Laura Lorenzo-López, Isabel González-Abraldes, Ana \\ Buján, Carmen de Labra and José Carlos Millánn-Calenti
}

Universidade da Coruña, Gerontology Research Group, Instituto de Investigación Biomédica de A Coruña (INIBIC) Complexo Hospitalario Universitario de A Coruña (CHUAC), SERGAS, Coruña, Spain

\begin{abstract}
Background: Multisensory stimulation and individualized music have shown to be good in handling the psychological and behavioral symptoms in people with severe dementia.

Objective: Explore the effects of two nonpharmacological interventions, multisensory stimulation environment (MSSE) in a Snoezelen room and individualized music sessions, on mood, behavior, and biomedical parameters of institutionalized elderly patients with severe dementia.

Methods: Randomized trial of 21 patients aged $\geq 65$ years randomly assigned to two groups (MSSE and individualized music). Interventions administered in two-weekly sessions lasted 30 minutes for a period of 12 weeks. Main outcomes were recorded before, during, and at the end of the intervention.

Results: Both groups had immediate positive effects on mood and behavior. Participants were more happy/more content $(p<0.001)$, talked more spontaneously $(p=0.009)$, related to people better $(p=0.002)$, were more attentive to/focused on their environment $(p<0.001)$, enjoyed themselves $(p=0.003)$, were less bored/inactive $(p=0.004)$, and more relaxed/content $(p=0.003)$. The MSSE group performed a better visual follow-up of the stimuli $(p=0.044)$, and the music group were more relaxed and happy $(\mathrm{p}=0.003)$. A decrease in heart rate $(\mathrm{p}=0.013)$ and an increase in oxygen saturation $(\mathrm{p}=0.011)$ were observed from before to after interventions in both groups, with no significant differences between them.

Conclusions: Both interventions seem to be effective at managing mood and behavioral disturbances in the short term and at improving physiological rates, highlighting the efficacy of nonpharmacological treatments in patients with severe dementia.
\end{abstract}

Keywords: Dementia, elderly, individualized music, randomized trial, Snoezelen 


\section{INTRODUCTION}

Dementia is one of the most important public health problems that our society faces. Its high prevalence in the elderly, along with the lack of effective treatments and the high degree of functional and cognitive dependence experienced by patients carries great medical, personal, familiar, social and economic impacts [1]. In advanced stages of dementia, patients show a variety of disrepair, including cognitive, functional, behavioral, and social decline, and also a wide range of neuropsychiatric symptoms [2], with dementia one of the main causes of disability in the elderly [3]. Traditionally, these behavioral and mood disturbances have been managed with medication but these pharmacological approaches have shown limited efficacy and can have some important adverse effects [4]. That is why nonpharmacological treatments are used increasingly and can improve both neuropsychiatric symptoms of patients and quality of life of caregivers [5]. The classical nonpharmacological behavioral interventions are not suitable for patients with severe dementia because their verbal communication is generally impaired [6]; they need specific nonpharmacological therapies with an appropriate environmental structure and simulation [7], such as individualized music interventions and multisensory stimulation environment (MSSE).

Music intervention uses music, in a receptive or in a participate way ([8], see [9] for a review), to get multiple benefits such as promoting socialization, stimulating communication and verbal and non- verbal expression, and helping to recover memories by evoking autobiographical events [10]. Further- more, music intervention is a non-invasive and inexpensive intervention that could be easily offered in residential settings [11], in particular thanks to the development of an evidence-based protocol of individualized music for the management of agitation in people with dementia to be implemented in healthcare facilities $[12,13]$. Individualized music has been defined as music integrated into the person's life and is based on personal preference [14]. Music intervention reduces neuropsychiatric symptoms in people with dementia [15, 16], with positive effects in disruptive behavior and anxiety (see [9] for a review, [17]). The use of music in elderly patients has shown a decrease in respiratory rate and an increase in temperature and oxygen saturation $[18,19]$.

On the other hand, initially, MSSE was introduced as a nonpharmacological treatment for people with learning difficulties [20]; nowadays more and more collectives have taken this therapy. Since the beginning of the $1990 \mathrm{~s}$, application of MSSE has been extended to the care of people with dementia, and in the last decades, it is becoming increasingly popular [21, 22]. This therapy is usually carried out in a pleas- ant and relaxing space known as a "Snoezelen room" [23]. MSSE involves the stimulation of the five senses by the patient's exploration of an environment, following a non-directive and facilitative approach [22], in which many objects such as fiber-optic cables, aromatherapy, light effects, calming sounds, water columns of different colors, and textured balls for tactile stimulation among others, are included [7, 24]. MSSE can be modified introducing only a single stimulation modality (i.e., individualized music) or even pairing individual elements (music, aroma, colored water columns) [25]. MSSE has demonstrated to be an effective intervention in the management of behavior and mood in short-term in elderly with dementia in a moderate and severe stage, in addition to encouraging interaction and communication ([21, 26], see [9] and [27] for reviews). The equipment required for setting up multisensory stimulation in a Snoezelen room may be easily acquired but is a fairly expensive investment [28]. That is why it is necessary to demonstrate the benefits of MSSE in comparison with other nonpharmacological interventions. However, there is insufficient evidence about the increased effectiveness of multisensory stimulation compared to other one-to-one interventions [29, 30]. Additionally, some studies [31, 32] report effects of MSSE on biomedical parameters, such as heart rate, blood hemoglobin, or salivary cortisol.

Both music therapy and MSSE have shown to be good in handling the psychological and behavioral symptoms in people with severe dementia, but there is limited evidence demonstrating which is more effective. For that reason, it is necessary to carry out studies including these two therapies to distinguish the potential benefits of each other. 
Therefore, the main objective of the current study was to assess whether MSSE in a Snoezelen room is more effective than individualized music sessions in regards to the mood, behavior, and biomedical parameters of institutionalized elderly patients with severe dementia.

\section{MATERIALS AND METHODS}

Design

We conducted a randomized longitudinal trial where participants were stratified according to their cognitive status being afterward randomly assigned to one of the two study groups (MSSE and individualized music).

\section{Participants}

Patients were recruited from a specialized dementia Gerontological Complex sited in A Coruña (Spain), with capacity for 70 people in a day care- setting and 64 institutionalized people in a nursing home. 21 users fulfilled the inclusion criteria of having a diagnosis of dementia and the presence of severe or very severe cognitive decline (Global Deterioration Scale [33], GDS 6-7). Diagnosis of dementia had been made and entered into the medical records by a neurologist. GDS was applied by a clinical psychologist with experience in assessing people to assess severity of dementia: severe (GDS 6) or very severe (GDS 7) cognitive decline. The exclusion criteria were: the presence of hearing impairment or other sensory disorder that would adversely affect interactions with the multisensory stimulation objects (e.g., severe vision impairment) and be bedridden.

A computer-based random number generator was used to randomly allocate the participants into one of the two groups according to their GDS score. 11 subjects were assigned to individualized music group and 10 subjects were assigned to MSSE group. A control group was not included in this study because our previous work [6] had already shown better effects of MSSE in comparison with control or one-toone activity sessions.

Approval was obtained from the Ethics Commit- tee at the University of A Coruña and the study was in conformity with the principles embodied in the Declaration of Helsinki. Before beginning data collection, all participants' proxies were previously informed about the study. Informed consent was obtained from a key family member or legal representative of each older person.

\section{Procedure}

The MSSE group participated in one-to-one multisensory sessions in a Snoezelen room. This room included several elements such as alternating colors fiber-optic cables, two water bubble columns within 2 mirrors, a water bed, a rotating mirror ball with a color light projector, a video, an interactive projecting system, musical selections, aromatherapy equipment with fragrant oils, and a tactile board with various textures, among others. In this group, the intervention followed the characteristics that define the MSSE [29]: visual, auditory, tactile, and olfactory stimulation was offered to patients; the therapists adopted a non-directive, enabling approach, encouraging patients to engage with sensory stimuli of their choice; and the stimuli used were non-sequential and unpatterned, experienced moment by moment with- out relying on short-term memory to link them to previous events. 
The individualized music group participated in music sessions according to their musical preferences. The intervention, including the main specifications of the guideline for individualized music proposed by Gerdner [12], occurred in a quiet room of the center, away from others. Subjects were familiarized with the room used for the intervention, which was also used by the professional staff for routine individual assessments and interventions, avoiding the agitation that could imply an unknown location. Each session of music intervention was presented "free field" on a computer and the volume or loudness of music was set at an appropriate level for each participant. In this group, the therapist (one for each session) followed a directive approach, selecting the music for each session, taking into account the preferences and interests of the participants.

Participants from both groups took part in two weekly sessions, for a period of 12 weeks, until they completed 24 sessions. Sessions lasted 30 minutes unless the participant expressed a desire to leave or if the patient exhibited a situation of increased agitation or confusion. In both groups the sessions followed an internal structure that involved an introduction to the session, carrying the session through, and winding the session down. However, in the MSSE group, there was some flexibility within the standardization, to address the singularity and individual needs/preferences of each patient, keeping with the traditional philosophy of multisensory stimulation.

The sessions were conducted by professionals in the field of psychology or occupational therapy, with equivalent education and training in the method- ology used. To avoid the creation of positive or negative expectations, the MSSE and the individualized music sessions were presented to the staff and caregivers as two equally valid interventions. As a result of this design, the differences found between the two conditions could be specifically attributed to the multisensory stimulation rather than more general therapeutic effects, such as the one-to-one attention to the patients. The data collection and administration of the intervention were carried out by the same therapist.

Data on the participants' sensorial preferences and interests were previously collected to design the con- tent of the sessions and minimize the behavioral problems that some participants could present within the MSSE and the music contexts. In the MSSE group, sensorial preferences in the Snoezelen room were assessed based on the procedure suggested by Pace et al. [34]. In the individualized music group, the significance of music prior to the patient's onset of cognitive impairment was determined. Family members knowledgeable about the patient's music preference were interviewed to get information as specific as possible. The Assessment of Personal Music Preference (APMPQ) (family version) [35] was used to assist in the selection of their family member's music preference. This instrument has been developed and tested [36] to obtain detailed information regarding personal music preferences and to identify the importance of music in the person's life during her or his independent living. It comprises a series of questions about the favorite types of music, forms of music, favorite artists/performs, and specific song titles prior to the onset of the cognitive impairment. The family version of APMPQ is used when the participants are unable to answer the questions due to cognitive impairment. This version has been successfully used by family members of residents living in long-term care facilities [36]. In our study, some items were revised to include types of Spanish music to make this assessment tool relevant to collect information regarding music preferences of older adults in Spain. The entire list of preferred music by each participant was stored in MP3 format on the PC used for intervention.

\section{Mood and behavior}

The participants' mood and behavior were rated before (10 minutes immediately before), during and after (10 minutes immediately after) the MSSE and individualized music sessions using the Interact scale [37]. Interact is a rating scale developed specifically for evaluating the effects of MSSE in dementia care. An inter-rater reliability of $r=0.99$ was found on a small sample [38]. In this study, 'Interact during' and 'Interact short' scales were used. 'Interact during' had a total of 22 items measured on a Likert scale and was scored according to the frequency of occurrence of each behavior, ranging from 1 (not at all) to 5 (nearly all the time). These data give an indication of the processes that occur within sessions. 
'Interact short', a 12-item version of Interact, was used to record mood and behavior during the 10 minutes immediately before sessions and the 10 minutes immediately after sessions to measure any observable changes. A Likert scale was applied to each item ranging from 1 (not at all) to 5 (nearly all the time). This gives an indication of the amount of change that each session produces in the short term.

Therapists received training in the use of the Interact scale, rated the same participants and discussed discrepancies. In order to avoid behavioral changes due to social desirability effect, Interact short was administered by therapists who work with the participants daily.

\section{Biomedical parameters}

Two biomedical parameters, heart rate (beats per minute) and oxygen saturation $\left(\mathrm{SpO}_{2}\right)$, were recorded immediately before and after sessions in the MSSE and individualized music groups using mobile finger pulse oximeters (Riester, Germany).

\section{Statistical analysis}

The sample characteristics were summarized as frequencies and percentages for the categorical variables and as the means and standard deviations for the continuous variables. We used the ShapiroWilk test to evaluate the normality of the sample. This test is more appropriate for small sample sizes ( $<50$ individuals).

Between-group comparisons were made using Chi-square test to test categorical variables and Student $t$ - test for continuous variables.

The immediate effects of the MSSE and individualized music sessions on patients' mood and behavior as measured by 'Interact short' were analyzed using paired $t$-tests to compare the means of scores from before sessions to the means of scores after sessions for each of the 12 outcome measurements. Within each group, Cohen's d values were reported as indicators of effect size (ES) for comparing the mean values. We interpreted the importance of the ES using the benchmarks for "small ES" $(d=0.2)$, "medium ES" $(d=0.5)$, and "large ES" $(d=0.8)$ as defined by Cohen [39].

Differences in the mood and behavior of patients during the MSSE and individualized music sessions as measured by 'Interact during' were analyzed using unpaired $t$-tests.

To determine whether there were any differences in the 'Interact short' scores from before to after a session between the groups, a repeated-measures two-way analysis of variance (two-way mixed ANOVAs) was conducted. The within-participants variable was the difference in measurements over time (before versus after), and the between- participants variable was the group (MSSE versus individualized music).

In addition, a repeated-measures two-way mixed ANOVA was used to determine whether there were any differences between the groups in the number of changes in biomedical parameters (heart rate and $\mathrm{SpO}_{2}$ ) from before to after the sessions. The within-participants variable was the measure over time (before and after), and the between-participants variable was the group (MSSE versus individualized music).

Eta-squared values $(\eta 2)$ were reported as indicators of effect size. Based on Cohen's recommendations [39], $\eta 2$ of $0.02,0.13$ and 0.26 indicate small, medium, and large effect sizes respectively. In all analysis, a $p$ value of less than 0.05 was considered statistically significant. Statistical analyses were con- ducted using the IBM SPSS Statistics v.23.0 (IBM Corp., Armonk, NY, USA). 


\section{RESULTS}

\section{Effects on mood and behavior}

The baseline sociodemographic characteristics of the residents at week 0 can be found in Table 1 . The mean age of the sample was 88.9 years (SD \pm 6.69 ), $71.4 \%$ were women, $71.4 \%$ of the patients were widowed, and $33.3 \%$ had secondary education. The MSSE and the individualized music groups were homogeneous in age, gender, marital status, and educational level.

Table 1. Sociodemographic characteristics of the residents with dementia at week 0 (Baseline, Pretrial) ${ }^{\mathrm{a}}$

\begin{tabular}{|c|c|c|c|c|}
\hline & $\operatorname{MSSE}(n=10)$ & Music $(n=11)$ & Total $(n=21)$ & $P$ \\
\hline \multicolumn{5}{|l|}{ Age (y) } \\
\hline Age range & $81-102$ & $77-97$ & $77-102$ & \\
\hline \multicolumn{5}{|l|}{ Gender, $n(\%)$} \\
\hline Female & $6(60.0)$ & $9(81.8)$ & $15(71.4)$ & 0.269 \\
\hline Single & $2(20.0)$ & $2(18.2)$ & $4(19.0)$ & 0.465 \\
\hline Married/partner & $1(10.0)$ & $0(0)$ & $1(4.8)$ & \\
\hline Widowed & $6(60.0)$ & $9(81.8)$ & $15(71.4)$ & \\
\hline Separated/divorced & $1(10.0)$ & $0(0)$ & $1(4.8)$ & \\
\hline \multicolumn{5}{|l|}{ Educational level, $n(\%)$} \\
\hline No formal education & $2(20.0)$ & $3(27.3)$ & $5(23.8)$ & 0.912 \\
\hline
\end{tabular}

MSSE, multisensory stimulation environment group; SD, standard deviation.

${ }^{\text {a }}$ Significance: $p$-value $<0.05$

Table 2 shows the means and SDs for each group on each item of Interact short and the results of paired $t$-tests. Significant improvements were observed from before sessions to after sessions in the MSSE group in the following items: more happy/content $(p=0.001)$, related to people better $(p=0.023)$, more attentive/focused on their environment $(p=0.005)$, enjoying themselves, more active or alert $(\mathrm{p}=$ $0.017)$, less bored/inactive $(p=0.026)$, and more relaxed, content, or sleeping appropriately $(p=0.021)$. The individualized music group showed significant improvements from before sessions to after sessions in the following items: more happy/content $(p=0.013)$, related to people better $(p=0.034)$, and more attentive/focused on their environment $(p=0.007)$. 
Table 2. Means scores (SDs) for each group (MSSE, $n=10$ versus Music, $n=11$ ) on INTERACT SHORT (before and after sessions)

\begin{tabular}{|c|c|c|c|c|c|c|}
\hline Construct & Item & Group & Before & After & $p$ & $d$ \\
\hline \multirow[t]{6}{*}{ Mood } & \multirow[t]{2}{*}{ Tearful/sad } & MSSE & $1.19(0.34)$ & $1.14(0.22)$ & 0.395 & 0.17 \\
\hline & & Music & $1.12(0.09)$ & $1.08(0.07)$ & 0.122 & 0.50 \\
\hline & Happy/content & Music & $2.65(0.55)$ & $2.85(0.46)$ & $0.013 *$ & -0.39 \\
\hline & \multirow{2}{*}{ Fearful/anxious } & MSSE & $1.21(0.44)$ & $1.13(0.19)$ & 0.364 & 0.24 \\
\hline & & Music & $1.06(0.10)$ & $1.10(0.18)$ & 0.533 & -0.27 \\
\hline & Confused & Music & $1.21(0.17)$ & $1.18(0.12)$ & 0.546 & 0.20 \\
\hline \multirow[t]{2}{*}{ Speech } & \multirow[t]{2}{*}{ Talked spontaneously } & MSSE & $1.52(0.71)$ & $1.74(0.81)$ & 0.052 & -0.29 \\
\hline & & Music & $1.50(0.52)$ & $1.71(0.66)$ & 0.084 & -0.35 \\
\hline \multirow[t]{2}{*}{ Relating to people } & \multirow[t]{2}{*}{ Related well } & MSSE & $2.16(0.66)$ & $2.45(0.83)$ & $0.023 *$ & -0.39 \\
\hline & & Music & $1.98(0.75)$ & $2.13(0.79)$ & $0.034 *$ & -0.19 \\
\hline Relating to environment & Attentive/focused on environment/objects & MSSE & $2.44(0.77)$ & $2.91(0.97)$ & $0.005 *$ & -0.54 \\
\hline \multirow{7}{*}{ Stimulation level } & Wandering, restless or aggressive & Music & $1.08(0.10)$ & $1.09(0.22)$ & 0.800 & -0.06 \\
\hline & \multirow[t]{2}{*}{ Enjoying self, active or alert } & MSSE & $1.75(0.59)$ & $2.13(0.81)$ & $0.017 *$ & -0.54 \\
\hline & & Music & $1.76(0.63)$ & $1.86(0.62)$ & 0.136 & -0.16 \\
\hline & \multirow[t]{2}{*}{ Bored, inactive or sleeping inappropriately } & MSSE & $2.91(0.68)$ & $2.42(0.89)$ & $0.026 *$ & 0.62 \\
\hline & & Music & $2.79(0.84)$ & $2.59(0.98)$ & 0.094 & 0.22 \\
\hline & \multirow[t]{2}{*}{ Relaxed, content or sleeping appropriately } & MSSE & $2.21(0.41)$ & $2.60(0.55)$ & $0.021 *$ & -0.80 \\
\hline & & Music & $2.55(0.45)$ & $2.75(0.59)$ & 0.100 & -0.38 \\
\hline
\end{tabular}

MSSE, multisensory stimulation group; * Significant $(p$-value $)<0.05$; d, effect size.

With regard to the repeated-measure ANOVA results, there were no significant differences between the groups from before to after sessions (group-time interactions).

Furthermore, there were significant time effects in 7 of the outcome measures: happy/content, talked spontaneously, related well, attentive to/focused on their environment, enjoying self, bored/inactive, and relaxed/content.

Participants in both groups were more happy/ content $\left(F_{(1,19)}=30.961, p<0.001\right)$, talked more spontaneously $\left(F_{(1,19)}=8.417, p=0.009\right)$, related to people better $\left(F_{(1,19)}=13.470, p=0.002\right)$, more attentive to/focused on their environment $\left(F_{(1,19)}=25.402, p<0.001\right)$, enjoying themselves $\left(F_{(1,19)}=\right.$ $11.825, p=0.003)$, less bored/inactive $\left(F_{(1,19)}=10.932, p=0.004\right)$, and more relaxed/content $\left(F_{(1,19)}=\right.$ $11.189, p=0.003)$, in the 10 minutes after the sessions compared to the 10 minutes before the sessions.

Regarding Interact during, there were differences between the groups during sessions in 'tracking observable stimuli' (Fig. 1) and in how 'relaxed/content' (Fig. 2) participants were. In the first case, the MSSE group was rated as more observant $(p=0.044)$ than the music group; while in the second one, music group participants were rated as more relaxed $(p=0.003)$ than MSSE group participants. 


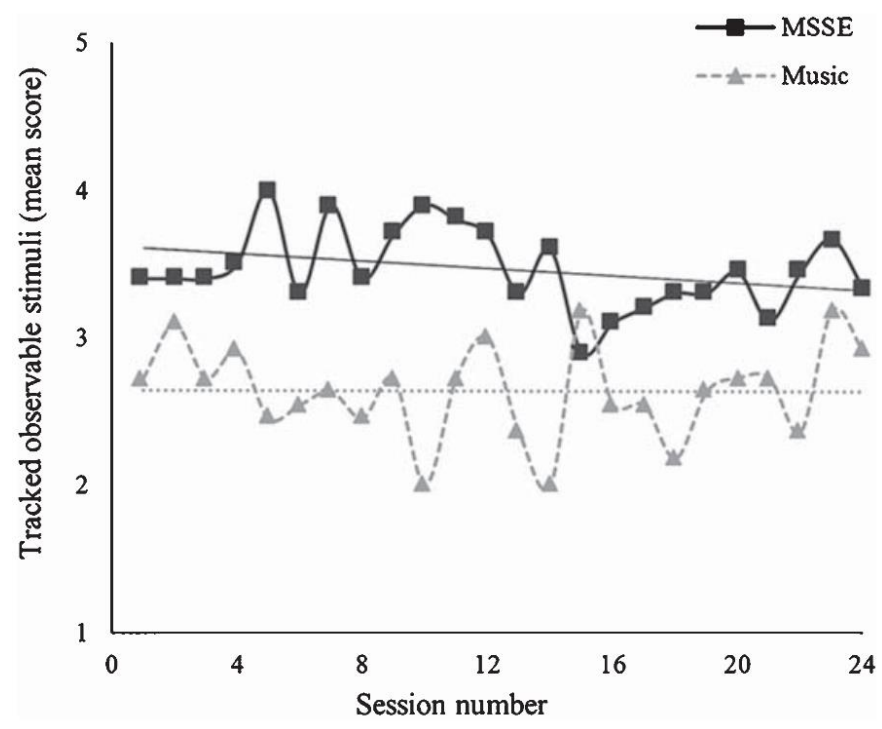

Fig. 1. Tracked observable stimuli. Interact during measurements at each session in two types of interventions-Multisensory Stimulation Environment (MSSE) and Individualized music - at sessions 0 (baseline) to 24 (post-trial). NOTE: $1=$ Not at all, $2=\mathrm{A}$ bit of the time, $3=$ Some of the time, $4=$ Most of the time, $5=$ Nearly all of the time.

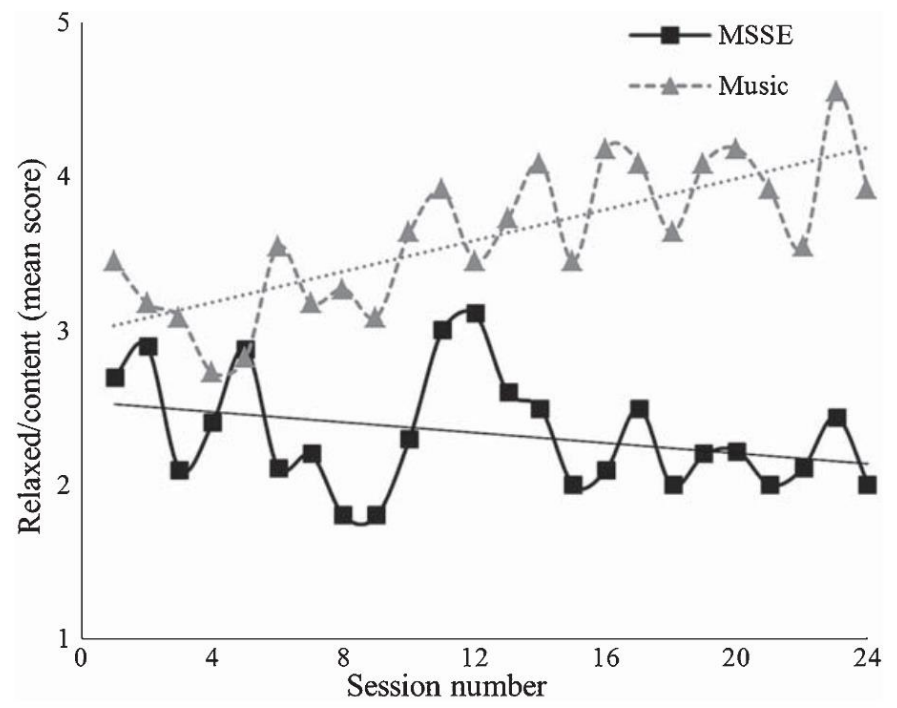

Fig. 2. Relaxed, content or sleeping appropriately. Interact during measurements at each session in two types of interventionsMultisensory Stimulation Environment (MSSE) and Individualized music - at sessions 0 (baseline) to 24 (post-trial). NOTE: $1=$ Not at all, $2=\mathrm{A}$ bit of the time, $3=$ Some of the time, $4=$ Most of the time, $5=$ Nearly all of the time 


\section{Effects on biomedical parameters}

Regarding biomedical parameters, there were significant time effects on heart rate (Fig. 3). Both groups reflected a decrease in heart rate from before to after sessions $\left(F_{(1,19)}=7.577, p=0.013\right)$, although no significant differences were found between the groups. Significant time effects were also found in $\mathrm{SpO}_{2}$ (Fig. 4). There was an increase in the mean $\mathrm{SpO}_{2}$ values of both groups from before to after the sessions $\left(F_{(1,19)}=8.025, p=0.011\right)$, with no significant differences between the groups.
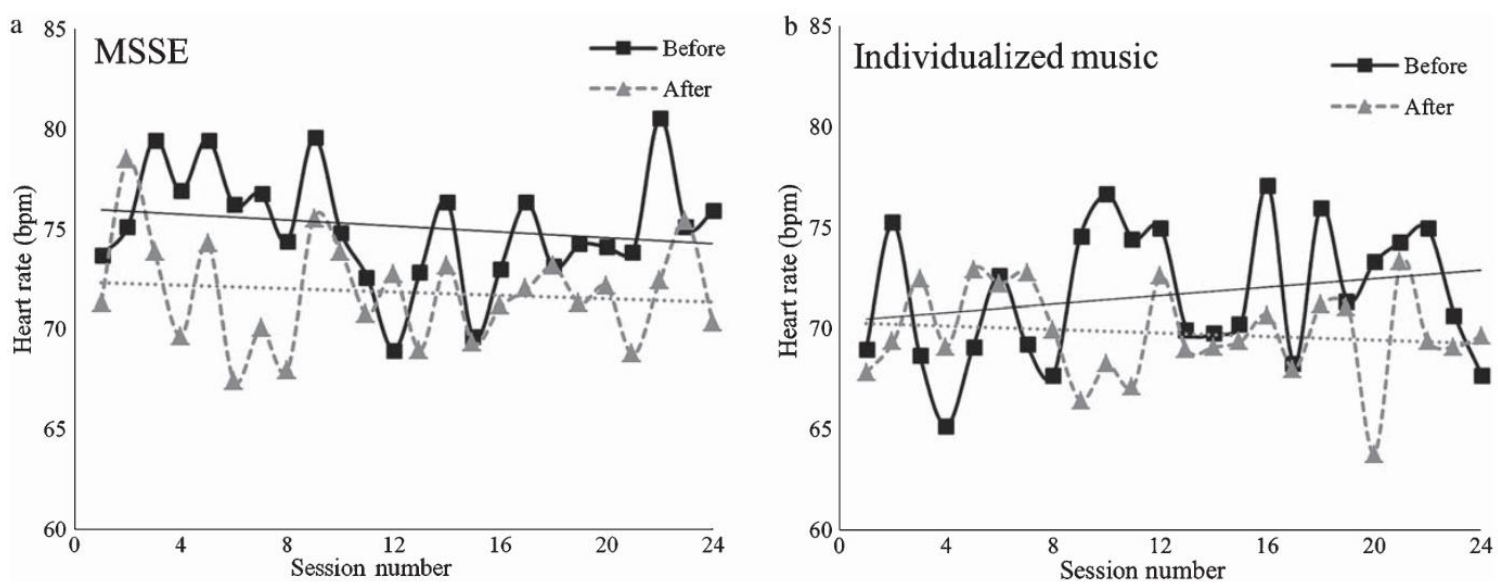

Fig. 3. Heart rate (beats per minute, bpm) before and after completion of two types of intervention-(a) Multisensory Stimulation Environment (MSSE) and (b) Individualized music —at sessions 0 (baseline) to 24 (post-trial).
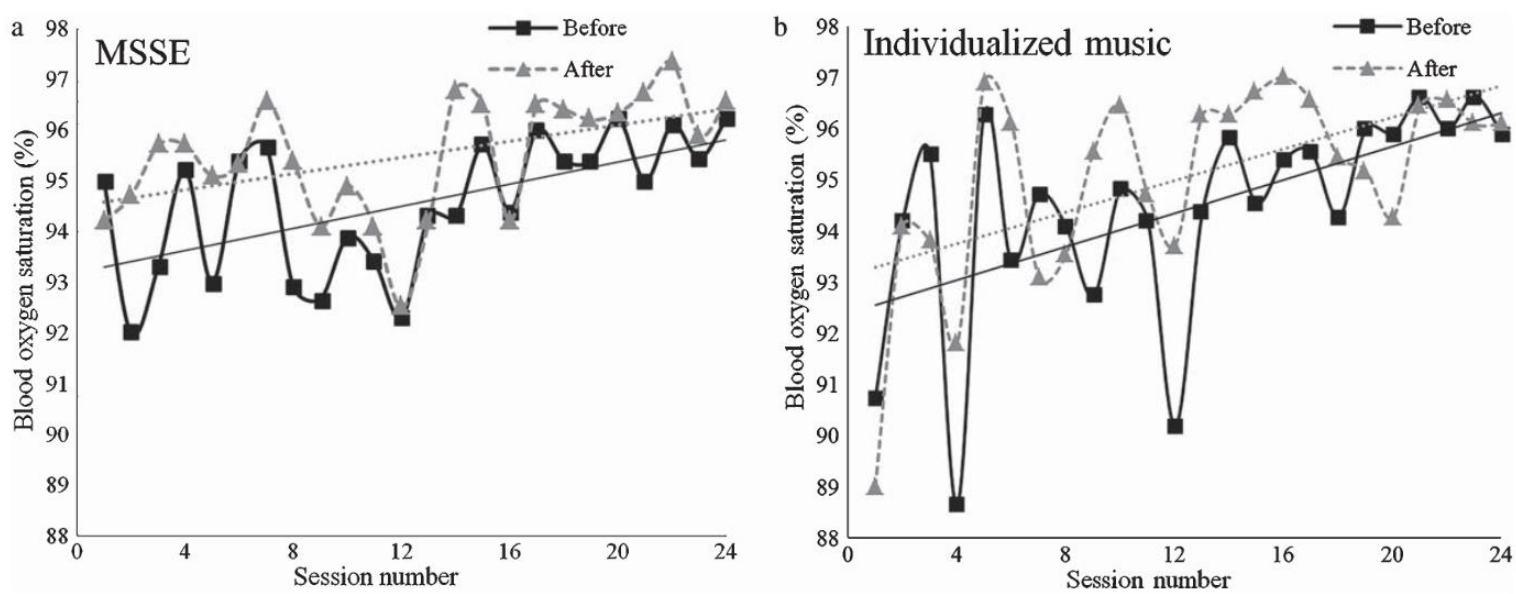

Fig. 4. Blood hemoglobin oxygen saturation (\%) before and after completion of two types of intervention-(a) multisensory stimulation environment (MSSE) and (b) Individualized music — at sessions 0 (baseline) to 24 (post-trial). 


\section{DISCUSSION}

\section{Effects of the type of intervention on mood and behavior}

\section{Interact short}

Results showed no significant differences between both the MSSE and the individualized music sessions when comparing the 10 minutes after the sessions with the 10 minutes before the sessions. However, both groups had immediate positive effects on mood and behavior. Participants in both groups were more happy/content, talked more spontaneously, related to people better, were more attentive to/focused on their environment, enjoyed themselves, were less bored/inactive and more relaxed/content from before to after sessions. Two theories, namely the Kovach Model of Imbalance in Sensoristasis [40] and the Functional Analytic Multisensory Environ- mental Therapy (FAMSET) [25], could explain what occurs during sensory stimulation in our participants. The first one [40], from a neurobiological perspective, postulates the need of pacing of sensory- stimulating and sensory-calming activities in persons with dementia to avoid intrapsychic discomfort. Multisensory stimulation ameliorates such negative consequences of imbalances in sensoristasis, providing stress-free and calming activities. On the other hand, the FAMSET theory [25] establishes that multisensory stimulation evokes states of reward and relaxation responses to reduce stress situations and to evoke well-being in people with dementia. Therefore, both interventions seem to be effective at managing mood and behavioral disturbances at the short term. Similar results were found in other studies [1, 31, 41] comparing MSSE in a Snoezelen room with other interventions, MSSE showed significant improvements in behavior immediately after the intervention but MSSE had no advantage over the other treatments. On the other hand, a study concluded that institutionalized people with dementia treated with MSSE had a significantly higher improvement in some of the neuropsychiatric symptoms than those who attended one-to-one activity sessions [42]. With similar findings, intervention with MSSE in a Snoezelen room improved significantly agitation levels compared to other recreational interventions [43]. A group of authors published a set of articles [44-47] showing that MSSE significantly improved mood and behavior of patients with moderate to severe dementia [44], and also the implementation of MSSE induced to a better quality of nurse-patient communication and better attendance in psychogeriatric care [45-47]. Regarding music intervention, several studies [48-54] evidenced the positive effects on the mood and behavior in patients with mild, moderate, or severe dementia after receiving individualized music sessions. However, previous studies $[55,56]$ have found opposite results, in which participation in music sessions did not improve levels of anxiety, depression, and agitation in older people with dementia. This lack of evidence may be reflective of measures coming from different respondents (carers/proxies versus the person with dementia) that would imply different results [55], or from the use of instruments with low sensitivity or randomization of participants without considering cognitive impairment level [56]. Another study [57] also concluded that individualized music did not have a significant effect on the behavioral and psychological symptoms of dementia (BPSD) in persons with moderate to severe dementia when compared with standard care received in their nursing homes. These authors explained the absence of statistically significant differences because the large number of participants who dropped out and the too small number of individualized music sessions.

Both interventions, MSSE and individualized music sessions, have shown some evidence for shortterm improvement in mood and behavioral disturbances, but their long-term effectiveness has not been proven enough $[21,58]$.

\section{Interact during}

Regarding mood and behavior throughout the sessions, we found significant differences between the MSSE and music individualized groups in only two of the items analyzed with the Interact during scale: 'tracking observable stimuli' and 'relaxed/content'. Participants in the MSSE group performed a better visual follow-up of the stimuli, and participants in the music group were more relaxed and happy compared to participants in the MSSE group. Associated with the first point, other authors have found that visual sense is most likely to be stimulated in a MSSE [27]. With respect to the second aspect, unlike 
our results, a study [23] evaluating the effectiveness of MSSE in comparison to individualized music sessions, showed a positive effect on anxiety-related symptoms in people with dementia during MSSE intervention that was not shown in the participants of the music group. Besides, in a case study [59] with three subjects, a high level of looking was obtained during Snoezelen sessions, but not in music sessions. In contrast to previous findings [29, 30], no significant effects on mood and behavior during MSSE sessions in comparison to other activity sessions were found. In terms of music intervention in older adults with dementia, some studies [59-63] demonstrated a reduction of agitated behaviors either active (based on singing, dancing or playing instruments) or passive forms (based on listening to music) of music intervention. In addition, interactive music intervention results in a greater improvement in participant's mood and BPSD comparing to passive music intervention [64].

\section{Effects on biomedical parameters}

A decrease in heart rate and an increase in oxygen saturation were observed in MSSE and music sessions from before to after interventions, with no significant differences between groups. This finding is consistent with previous studies [31, 65] in which no significant different effects of the MSSE com- pared to other one-to-one activities on the biomedical parameters in patients with mild, moderate or severe dementia, were found. However, a study analyzing heart rate and respiration in patients in the final stage of dementia, showed distinct reactions to music than to touch or object presentation [66].

\section{Limitations and recommendations for future research}

Several limitations should be noted when interpreting our findings. First, the relatively small sample size, with 11 individuals included in each group, which may account the no significant results found in some of the measured outcomes. Notably, it should be considered the difficulty of obtaining this specific type of older individuals with severe or very severe dementia for ensuring the homogeneity (same baseline characteristics) and randomization of both intervention groups. Future studies should address this limitation by including larger samples to confirm our findings. Second, the great economic investment that entails the use of a Snoezelen room compared to other therapies for people with dementia. Therefore, it is highly important to demonstrate in an empirical way that the benefits of MSSE in a Snoezelen room on mood and behavioral disturbances of people with severe dementia are better or greater than those provided by other sensory interventions that require minor economic resources such as music intervention or light therapy [7], especially since individualized music can be effectively implemented by nursing assistants, activity staff, or family members in a variety of settings [67].

\section{Conclusions}

This study evidences that both MSSE sessions and individualized music sessions are effective nonpharmacological treatments for the management of BPSD in people with severe dementia. MSSE sessions in Snoezelen room were found to be as effective as individualized music sessions, except during the intervention sessions, with differences in two of the analyzed parameters: 'tracking observable stimuli' and 'relaxed/content'; which means that participants in the MSSE group performed a better visual followup of the stimuli than the participants in the individualized music group, while participants in the music group were more relaxed and happy than those of the MSSE group. Regarding physiological rates, both groups exhibited an improvement in heart rate and oxygen saturation from before to after the sessions. Future empirical studies are needed to con- firm our results and to examine the benefits of the MSSE in a room Snoezelen versus another type of interventions. 


\section{ACKNOWLEDGMENTS}

This work was supported by the Xunta de Galicia (ED431C 2017/49 and FrailNet network IN607C, 2016/08). We thank the users and staff of the Gerontology Complex La Milagrosa, without whom this study would not have been possible. We are truly grateful to Prof Roger Baker for providing us with a copy of and information about the Interact scale.

Authors' disclosures available online (https:// www.j-alz.com/manuscript-disclosures/18-0109r1).

\section{REFERENCES}

[1] Castellanos Pinedo F, Cid Gala M, Duque San Juan P, Zurdo M (2011) Abordaje integral de la demencia. Inf Ter Sist Nac Salud. 35, 39-45.

[2] Koopmans RTCM, van der Molen M, Raats M, Ettema TP (2009) Neuropsychiatric symptoms and quality of life in patients in the final phase of dementia. Int J Geriatr Psychiatry. 24, 25-32.

[3] Jorm AF (1994) Disability in dementia: Assessment, prevention, and rehabilitation. Disabil Rehabil. 16, 98-109.

[4] Thornley J, Hirjee H, Vasudev A (2016) Music therapy in patients with dementia and behavioral disturbance on an inpatient psychiatry unit: Results from a pilot randomized controlled study. Int Psychogeriatr. 28, 869-871.

[5] Olazarán J, Reisberg B, Clare L, Cruz I, Peña-Casanova J, Del Ser T, Woods B, Beck C, Auer S, Lai C, Spector A, Fazio S, Bond J, Kivipelto M, Brodaty H, Rojo JM, Collins H, Teri L, Mittelman M, Orrell M, Feldman HH, Muñiz R (2010). Nonpharmacological therapies in Alzheimer's disease: A systematic review of efficacy. Dement Geriatr Cogn Disord. 30, 161178.

[6] Sánchez A, Marante-Moar MP, Sarabia C, de Labra C, Lorenzo T, Maseda A, Millán-Calenti JC (2016) Multisensory stimulation as an intervention strategy for elderly patients with severe dementia: A pilot randomized controlled trial. Am J Alzheimers Dis Other Demen. 31, 341-350.

[7] Behrman S, Chouliaras L, Ebmeier KP (2014) Considering the senses in the diagnosis and management of dementia. Maturitas. 77, 305-310.

[8] Berger G, Bernhardt T, Schramm U, Müller R, LandsiedelAnders S, Peters J, Kratzsch T, Frolich L (2004) No effects of a combination of caregivers support group and memory training/music therapy in dementia patients from a memory clinic population. Int J Geriatr Psychiatry. 19, 223-231.

[9] Abraha I, Rimland JM, Trotta FM, Dell'Aquila G, Cruz-Jentoft A, Petrovic M, Gudmundsson A, Soiza R, O'Mahony D, Guaita A, Cherubini A (2017) Systematic review of systematic reviews of non-pharmacological interventions to treat behavioural disturbances in older patients with dementia. The SENATOR-OnTop series. BMJ Open. 7,e012759.

[10] Cammisuli DM, Danti S, Bosinelli F, Cipriani G (2016) Non-pharmacological interventions for people with Alzheimer's Disease: A critical review of the scientific literature from the last ten years. Eur Geriatr Med. 7, 57-64.

[11] Blackburn R, Bradshaw T (2014) Music therapy for service users with dementia: A critical review of the literature. J Psychiatr Ment Health Nurs. 21, 879-888.

[12] Gerdner LA (2013) Evidence-Based Guideline: Individualized Music for Persons with Dementia. 5th ed., Series on Evidence-Based Practice for Older Adults (series editor, Titler M). National Nursing Practice Network, University of Michigan, School of Nursing: Ann Arbor, Michigan.

[13] Gerdner LA, Buckwalter KC (2017) Clarification: Research and associated evidence-based protocol for individualized music in persons with dementia. Am J Geriatr Psychiatry 25, 12891291.

[14] Gerdner LA (1992) The effects of individualized music on elderly patients who are confused and agitated. Master's thesis, University of Iowa, Iowa City.

[15] Satoh M, Yuba T, Tabei K, Okubo Y, Kida H, Sakuma H, Tomimoto H (2015) Music therapy using singing training improves psychomotor speed in patients with Alzheimer's disease: A neuropsychological and fMRI study. Dement Geriatr Cogn Dis Extra 5, 296-308.

[16] Millán-Calenti JC, Lorenzo-López L, Alonso Búa B, de Labra C, González-Abraldes I, Maseda A (2016) Optimal non-pharmacological management of agitation in Alzheimer's disease: Challenges and solutions. Clin Interv Aging 11, 175-184. 
[17] Zhang Y, Cai J, An L, Hui F, Ren T, Ma H, Zhao Q (2017) Does music therapy enhance behavioral and cognitive function in elderly dementia patients? A systematic review and metaanalysis. Ageing Res Rev 35, 1-11.

[18] Lin YJ, Lu KC, Chen CM, Chang CC (2012) The effects of music as therapy on the overall well-being of elderly patients on maintenance hemodialysis. Biol Res Nurs 14, 277-285.

[19] Geethanjali B, Adalarasu K, Jagannath M, Rajasekaran R (2016) Influence of pleasant and unpleasant music on cardiovascular measures and task performance. Int J Biomed Eng Technol 21, 128-144.

[20] Burns I, Cox H, Plant H (2000) Leisure or therapeutics? Snoezelen and the care of older persons with dementia. Int $J$ Nurs Pract 6, 118-126.

[21] Sánchez A, Millán-Calenti JC, Lorenzo-López L, Maseda A (2013) Multisensory stimulation for people with dementia: A review of the literature. Am J Alzheimers Dis Other Demen 28, 7 14

[22] López-Almela A, Gómez-Conesa A (2011) Intervención en demencias mediante estimulación multisensorial (snoezeen). Fisioterapia 33, 79-88.

[23] Sánchez A, Maseda A, Marante-Moar MP, de Labra C, Lorenzo-López L, Millán-Calenti JC (2016) Comparing the effects of multisensory stimulation and individualized music sessions on elderly people with severe dementia: A randomized controlled trial. J Alzheimers Dis 52,303315.

[24] Milev RV, Kellar T, McLean M, Mileva V, Luthra V, Thompson S, Peever L (2008) Multisensory stimulation for elderly with dementia: A 24-week single-blind randomized controlled pilot study. Am J Alzheimers Dis Other Demen 23,372-376.

[25] Staal J (2012) Functional analytic multisensory environmental therapy for people with dementia. Int J Alzheimers Dis 2012, 294801.

[26] Berkheimer SD, Qian C, Malmstrom TK (2017) Snoezelen therapy as an intervention to reduce agitation in nursing home patients with dementia: A pilot study. J Am Med Dir Assoc 18, 10891091.

[27] Collier L, Jakob A (2017) The Multisensory Environment (MSE) in dementia care: Examining its role and quality from a user perspective. HERD 10, 39-51.

[28] Lancioni GE, Cuvo AJ, O'Reilly MF (2002) Snoezelen: An overview of research with people with developmental disabilities and dementia. Disabil Rehabil 24, 175-184.

[29] Baker R, Bell S, Baker E, Gibson S, Holloway J, Pearce R, Dowling Z, Thomas P, Assey J, Wareing LA (2001) A randomized controlled trial of the effects of multi-sensory stimulation (MSS) for people with dementia. Br J Clin Psychol 40, 81-96.

[30] Baker R, Holloway J, Holtkamp CCM, Larsson A, Hartman LC, Pearce R, Scherman B, Johansson S, Thomas PW, Wareing LA, Owens M (2003) Effects of multi-sensory stimulation for people with dementia. J Adv Nurs 43, 465-477.

[31] Baillon S, Van Diepen E, Prettyman R, Redman J, Rooke N, Campbell R (2004) A comparison of the effects of Snoezelen and reminiscence therapy on the agitated behaviour of patients with dementia. Int J Geriatr Psychiatry 19, 1047-1052.

[32] Chu H, Yang CY, Lin Y, Ou KL, Lee TY, O'Brien AP, Chou KR (2014) The impact of group music therapy on depression and cognition in elderly persons with dementia: A randomized controlled study. Biol Res Nurs 16, 209-217.

[33] Reisberg B, Ferris SH, de Leon MJ, Crook T (1982) The global deterioration scale for assessment of primary degenerative dementia. Am J Psychiatry 139, 1136-1139.

[34] Pace GM, Ivancic MT, Edwards GL, Iwata BA, Page TJ (1985) Assessment of stimulus preference and reinforce value with profoundly retarded individuals. J Appl Behav Anal 18, 249-255.

[35] Gerdner LA, Hartsock J, Buckwalter KC (2000) Assessment of personal music preference (family version). The University of Iowa Gerontological Nursing Interventions Research Center: Research Development and Dissemination Iowa City, Iowa, Core.

[36] Gerdner LA (2005) Use of individualized music by trained staff and family: Translating research into practice. J Gerontol Nurs 31, 22-30.

[37] Baker R, Dowling Z (1995) INTERACT. King's Park Community Hospital, Dorset Healthcare NHS Trust, Bournemouth.

[38] Wareing LA, Coleman PG, Baker R (1998) Multisensory environments and older people with dementia. Br J Ther Rehabil 5, 624-629.

[39] Cohen J (1988) Statistical power analysis for the behavioural sciences. 2nd ed. Erlbaum, Hillsdale.

[40] Kovach CR (2000) Sensoristasis and imbalance in persons with dementia. J Nurs Scholarsh 32, 379-384. 
[41] Bauer M, Rayner JA, Tang J, Koch S, While C, O’Keefe F (2015) An evaluation of Snoezelen ${ }^{\circledR}$ compared to 'common best practice' for allaying the symptoms of wandering and restlessness among residents with dementia in aged care facilities. Geriatr Nurs 36, 462-466.

[42] Maseda A, Sánchez A, Marante MP, González-Abraldes I, Buján A, Millán-Calenti JC (2014) Effects of multisensory stimulation on a sample of institutionalized elderly people with dementia diagnosis: A controlled longitudinal trial. Am J Alzheimers Dis Other Demen 29, 463 473.

[43] Staal J, Sacks A, Matheis R, Collier L, Calia T, Hanif H, Kofman ES (2007) The effects of Snoezelen (multi-sensory behavior therapy) and psychiatric care on agitation, apathy, and activities of daily living in dementia patients on a short term geriatric psychiatric inpatient unit. Int J Psychiatry 37,357-370.

[44] van Weert JCM, van Dulmen AM, Spreeuwenberg PMM, Ribbe MW, Bensing JM (2005) Behavioral and mood effects of Snoezelen integrated into 24-hour dementia care. J Am Geriatr Soc 53, 24-33.

[45] van Weert JCM, van Dulmen AM, Spreeuwenberg PMM, Ribbe MW, Bensing JM (2005) Effects of snoezelen, integrated in $24 \mathrm{~h}$ dementia care, on nurse-patient communication during morning care. Patient Educ Couns 58, 312-326.

[46] van Weert JCM, van Dulmen AM, Spreeuwenberg PMM, Bensing JM, Ribbe MW (2005) The effects of the implementation of snoezelen on the quality of working life in psychogeriatric care. Int Psychogeriatr 17, 407-427.

[47] van Weert JCM, Janssen BM, van Dulmen AM, Spreeuwenberg PM, Bensing JM, Ribbe MW (2006) Nursing assistants' behaviour during morning care: Effects of the implementation of snoezelen, integrated in 24-hour dementia care. $J$ Adv Nurs 53, 656-668.

[48] Gold K (2014) But does it do any good? Measuring the impact of music therapy on people with advanced dementia: (Innovative practice). Dementia (London) 13, 258-264.

[49] Guétin S, Portet F, Picot MC, Pommié C, Messaoudi M, Djabelkir L, Olsen AL, Cano MM, Lecourt E, Touchon J (2009) Effect of music therapy on anxiety and depression in patients with Alzheimer's type dementia: Randomised, controlled study. Dement Geriatr Cogn Disord 28, 36-46.

[50] Hsu MH, Flowerdew R, Parker M, Fachner J, Odell-Miller H (2015) Individual music therapy for managing neuropsychiatric symptoms for people with dementia and their carers: A cluster randomised controlled feasibility study. BMC Geriatrics 15,84 .

[51] Suzuki M, Kanamori M, Watanabe M, Nagasawa S, Kojima E, Ooshiro H, Nakahara D (2004) Behavioral and endocrinological evaluation of music therapy for elderly patients with dementia. Nurs Health Sci 6, 11-18.

[52] Svonsdottir HB, Snaedal J (2006) Music therapy in moderate and severe dementia of Alzheimer's type: A case-control study. Int Psychogeriatr 18, 613-621.

[53] Gerdner LA (1997) An individualized music intervention for agitation. J Am Psychiatr Nurs Assoc 3, 177-184.

[54] Gerdner LA (2000) Effects of individualized vs. classical "relaxation" music on the frequency of agitation in elderly persons with Alzheimer's disease and related disorders. Int Psychogeriatr $12,49-65$.

[55] Cooke ML, Moyle W, Shum DHK, Harrison SD, Murfield JE (2010) A randomized controlled trial exploring the effect of music on agitated behaviours and anxiety in older people with dementia. Aging Ment Health 14, 905-916.

[56] Vink AC, Zuidersma M, Boersma F, de Jonge P, Zuidema SU, Slaets JP (2013) The effect of music therapy compared with general recreational activities in reducing agitation in people with dementia: A randomised controlled trial. Int J Geriatr Psychiatry 28, 1031-1038.

[57] Raglio A, Bellandi D, Baiardi P, Gianotti M, Ubezio MC, Zanacchi E, Granieri E, Imbriani M, Stramba-Badiale M (2015) Effect of active music therapy and individualized listening to music on dementia: A multicenter randomized controlled trial. J Am Geriatr Soc 63, 1534-1539.

[58] McDermott O, Crellin N, Ridder HM, Orrell M (2013) Music therapy in dementia: A narrative synthesis systematic review. Int J Geriatr Psychiatry 28, 781-794.

[59] Pinkney L (1997) A comparison of the Snoezelen environment and a music relaxation group on the mood and behaviour of patients with senile dementia. Br J Occup Ther 60, 209-212.

[60] Goddaer J, Abraham IL (1994) Effects of relaxing music on agitation during meals among nursing home residents with severe cognitive impairment. Arch Psychiatr Nurs 8, 150-158.

[61] Tabloski PA, Mckinnon-Howe L, Remington R (1995) Effects of calming music on the level of agitation in cognitively impaired nursing home residents. Am J Alzheimers Dis Other Demen $10,10-15$.

[62] Clark ME, Lipe AW, Bilbrey M (1998) Use of music to decrease aggressive behaviors in people with dementia. J Gerontol Nurs 24, 10-17. 
[63] Ridder HMO, Stige B, Qvale LG, Gold C (2013) Individual music therapy for agitation in dementia: An exploratory randomized controlled trial. Aging Ment Health 17, 667-678.

[64] Sakamoto M, Ando H, Tsutou A (2013) Comparing the effects of different individualized music interventions for elderly individuals with severe dementia. Int Psychogeriatr 25, 775-784.

[65] Maseda A, Sánchez A, Marante MP, González-Abraldes I, de Labra C, Millán-Calenti JC (2014) Multisensory stimulation on mood, behavior, and biomedical parameters in people with dementia: Is it more effective than conventional one-to-one stimulation? Am J Alzheimers Dis Other Demen 29, 637-647.

[66] Norberg A, Melin E, Asplund K (2003) Reactions to music, touch and object presentation in the final stage of dementia: An exploratory study. Int J Nurs Stud 40, 473-479.

[67] Gerdner LA, Schoenfelder DP (2010) Evidence-based guideline. Individualized music for elders with dementia. J Gerontol Nurs 36, 7-15. 